\title{
New or noteworthy trees from Micronesia (III)
}

\author{
by
}

\section{Ryôzô Kanehira}

Received June 27, 1932

(30) Columbia Burreti Kanehira nom. nov.

Microcos scabra Sm. in Rees Cycl. XXIII (1819) No. 3.

Colona scabra Burret in Notizb. Bot. Berl. IX (1926) 800.

Columbia scabra (Sм.) KanehIra in Tokyo Bot. Mag. XLV (1931) 295, non Aug. DC. in Bull. Herb. Boiss. sér. II, iii, 368.

Palau Armatin 476 July 1929. In forests along the seashore.

Distrib. Celebes Amboina, Arue Islands. Nat. name: Uab (Palau).

\section{(31) Eugenia stelechanthoides Kanehira sp. nov.}

Jambosa stelechantha Diels in ENGL. Bot. Jahrb. LVI (1921) 533, pro parte., quod GibBons 1065. Species Eugenia stelechanthae affinis, differt ramis ramulisque quadrangularis distincte alatis, foliis majoribus.

A medium-sized tree $20-40 \mathrm{~cm}$. in diameter, 3-7 m. high, branches and branchlets quadrangular and distinctly winged. Leaves opposite, subsessile, coriaceous, olivaceous when dry, lanceolate up to $50 \mathrm{~cm}$. long, $12 \mathrm{~cm}$. wide, caudately acuminate, base subobtuse, unequilaterally cordate near the junction of petiole, lateral nerves about 18 on each side of the midrib, anastomosing, midrib and nerves prominently elevated underneath, reticulations distinct. Cymes terminal or fascicled on the trunk and older branches, glabrous, calyx obconical, irregularly dentate, $5 \mathrm{~mm}$. in diameter, $5 \mathrm{~mm}$. long, petals oblong, 3-4 $\mathrm{mm}$. long ; fruit red, globose, up to $1.5 \mathrm{~cm}$. in diameter.

Kusaian species is distinguished from Ponapean form by its winged branchlets and much larger leaves. This is Jambosa stelechantha in part as to the Kusaian material cited by Diess. Kusai Nos. 1318, 1348, 1431! July 1931 (The type in Herb. Forest. Inst., Kyushu Imperial Univ.). In forests from low to high altitudes. The tree is very common in Kusai, often occurring in groups. It is very conspicuous in the forests because of its white fragrant cauline flowers and brilliant red fruits clustered on the trunk. The tree is called " neis" by the natives.

Distrib. Endemic. 
(32) Medinilla diversifolia Kanehrra sp. nov.

Frutex erectus, $1 \mathrm{~m}$. altus, ramulis et petiolis et subtus foliis ad costam nervosque plus minusve furfuraceis, ramis ramulisque subteretibus, foliis petiolatis, oppositis, in paribus inaequalibus, ovato-oblongis ad ovatis, majoribus 6.5 ad $9 \mathrm{~cm}$. longis, ad $5 \mathrm{~cm}$. latis, minoribus $3-5 \mathrm{~cm}$. longis, $1.5-3 \mathrm{~cm}$. latis, 3-vel 5-plinerviis, reticulis nullis, apice leviter acuminatis, basi subrotundatis, petiolo $3-8 \mathrm{~mm}$. longo ; inflorescentiis lateralibus $7-9 \mathrm{~cm}$. longis, floribus verticellatis, bracteolis linearis, 5-10 $\mathrm{mm}$. longis, $2-4 \mathrm{~mm}$. latis; fructibus succulentis, ampulliformibus, roseis, 4-locellatis, circ. 10-12 mm. latis, bractis binis subtensis, bracteis accrescentiis, ovatis, roseis, usque ad $2 \mathrm{~cm}$. longis, 3-vel 5-plinerviis, pedicellis tenuibus, $10 \mathrm{~mm}$. longis.

A very handsome shrub, well characterized by its variable leaves. Kusai, in damp primary forests at the top of Mt. Hinkoln (alt. $630 \mathrm{~m}$.), No. 1371 ! July 1931 (The type in Herb. Forest Inst. Kyushu Imp. Univ.). Distrib. Endemic.

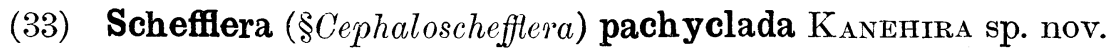

Arbor epiphytica circ. 6-8 m. alta, ramis ramulisque crassis, foliolis digitatis, 7-8, oblongo-lanceolatis, in siccitate olivaceo-nigrocantibus, 16$20 \mathrm{~cm}$, longis, $5-7 \mathrm{~cm}$. latis, coriaceis, glabris, apice obtusis ad breviter rostratis, basi subobtusis, costa supra planata vel sulcata, subtus elevata, nervis lateralibus circ. 10-12, indistinctis, reticulis obscuris, petiolis cylindraceis, $20-35 \mathrm{~cm}$. longis, petiolulis glabris $5-6 \mathrm{~cm}$. longis. Paniculis terminalibus elongatis, $50 \mathrm{~cm}$. vel ultra longis, glabris, pedunculis capitulorum racemose dispositis, glabris, $2-2,5 \mathrm{~cm}$. longis, capitulis circ. $10-$ floris, subglobosis, $1.5 \mathrm{~cm}$. diam., bracteis 5 , reniformibus, floris involucrum formatibus, inaequilibus, majoribus $4-5 \mathrm{~mm}$. latis, $5 \mathrm{~mm}$. longis, minoribus $3.5 \mathrm{~mm}$. latis, $2.5 \mathrm{~mm}$. longis, calycibus infundibuliformibus $2 \mathrm{~mm}$. longis, $2.5 \mathrm{~mm}$. latis margine integro, corollae calyptratae, oblongae 5-6 mm. longae $5 \mathrm{~mm}$. latis, apice subrotundatae, stamina 10, ad marginem disci insertis, filamentis glabris, $1 \mathrm{~mm}$. longis, antheris $2-3 \mathrm{~mm}$. longis, bilocularis, stylus subnullus, ovario crasso 10-vel 11-loculari, loculis 1-ovulatis; fructibus ovoideo-globosis, 6-7 mm. latis, 7-8 mm. longis, distincte 10-vel 11-sulcatis.

A medium-sized tree, usually branched from the bottom of the trunk. It is near Scheffera megalantha Harms from New Guinea but is distinguished by its much smaller fllowers.

In secondary forests, Truk Tol, alt. 300 m. No. 1272 June 1931; Truk Fefan, alt. 250 m. No. 1720! Aug. 1931 (The type in Herb. Forest Inst. Kyushu Imp. Univ.) 
Distrib. Endemic. Nat name: Ursyau (Truk).

(34) Sideroxylon micronesicum $\mathrm{K}_{\text {ANEHIRA }}$ sp. nov.

Arbor 7-8 m. alta, trunco $60-80 \mathrm{~cm}$. diametro, ramis ramulisque crassis, fusco-brunneis, minute lenticellatis; foliis membranaceis, ovatooblongis ad oblongis, usque ad $25 \mathrm{~cm}$. longis, $9 \mathrm{~cm}$. latis, utrinque glabris, in siccitate olivaceo-pallidis, nitidis, apice obtusis ad breviter acuminatis, basi obtusis ad cuneato-obtusis, leviter decurrentibus, nervis lateralibus circ. 16-18, utrinque prominentibus, anastomosantibus, reticulis distinctis, petiolo usque ad $5 \mathrm{~cm}$. longo, complanato. Floribus minimis $3 \mathrm{~mm}$. diametro, axillaribus vel e axillis defoliatis, pedicellis tenuibus, $5 \mathrm{~mm}$. longis, tomentosis, pedicellis basi bracteis numerosis subtensis, calycis segmentis $5,1.5 \mathrm{~mm}$. longis, extus parce tomentosis, corollae tubo quam lobis brevioribus, lobis 5 , orbicularis, $1.5 \mathrm{~mm}$. longis, stamina $5,0.7 \mathrm{~mm}$. longa, ovario ovoideo, carinato; fructibus ovoideis, $15 \mathrm{~mm}$. longis, $11 \mathrm{~mm}$. latis, glabris, apice subacutis, 3-vel 4-locularis.

A species characterized in its large glabrous leaves, very minute flowers and 3-or 4-celled fruits.

Kusai, in damp primary forests at medium and high altitudes, Nos. 1322 (fr.)!1462! (flos.) Julys 1931 (The types in Herb. Forst Inst. Kyusyu Imp. Univ.)

Distrib. Endemic. Nat. name: Sohs (Kusai).

(35) Canthium korrense (VAL.) Kanehira com. nov.

Plectronia korrensis VAL. in ENGL. Bot. Jahrb. LXIII (1930) 311.

Ponape: Nos. 1482, 1489, 1497, 1520, 1523, 1546, 1653, July 1931. In primary forests between Kolonia and Parkier at alt. 100-250 m.

Distrib. Endemic. The type from Ponape.

(36) Canthium oblongum (VAL.) Kanehira comb. nov.

Plectronia oblonga VAL. 1. c. 310.

Geniostoma niinoanense KanehIRA in Tokyo Bot. Mag. XLV (1931) 341.

Ponape 798! Niinoani-zan Aug. 1929, Parkier 1699 July 1931. In primary forests from medium to high altitudes.

Distrib. Endemic. The type from Ponape.

(37) Canthium Valetonii (VAL.) Kanehira Nom. nov.

Plectronia obovata VAL. 1. c. 311-non Canthium obovatum KLotzsch in ECKL. et ZEYH. Enum. (1834-37) 361.

Distrib. Endemic; the type from Ponape. 


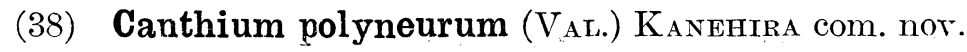

Plectronia polyneura VAc. 1. c. 309.

Distrib. Endemic; the type from Ponape.

(39) Canthium verticillatum (VAL.) Kanehira comb. nov.

Plectronia verticillata VAL. 1. c. 312.

Distrib. Endemic; the type from Palau.

(40) Mischocarpus Guillauminii Kanehrra sp. nov.

Arbor 5-6 m. alta, $50 \mathrm{~cm}$. diametro, ramis ramulisque teretibus, glabris vel glabrescentibus, foliis paripinnatis, alternis, $30 \mathrm{ad} 40 \mathrm{~cm}$. longis, longe petiolatis 2-vel 3 -jugis; foliolis oppositis, coriaceis, in siccitate olivaceo-pallidis, concoloribus, oblongo-lanceolatis, $15-22 \mathrm{~cm}$. longis, 5-8 $\mathrm{cm}$. latis, acuminatis, basi obtusis ad inaequilateraliter rotundatis, nervis primariis 8 ad 11, arcuato-anastomosantibus, reticulis utrinque perspicuis, petiolulis $5-10 \mathrm{~mm}$. longis; infructescentiis paniculatis, usque ad 20 $\mathrm{cm}$. longis, $12 \mathrm{~cm}$. latis; fructibus ovoideis, $2.5 \mathrm{~cm}$. longis, trilocularis, seminibus ovoideis, $18 \mathrm{~mm}$. longis, $12 \mathrm{~mm}$. latis, partibus inferioribus arillatis, arillis lutescentiis; floribus ignotis.

Truk Tol No. 1368! June 1931. (The type in Herb. Forest. Inst. Kyushu Imp. Univ.) In primary forests altitude about $450 \mathrm{~m}$.

The first representative of the genus to be recorded from the Carolines, although the known range of the genus extends from India to New Guinea, through Sumatra, Cochin China, Philippines and Australia.

Named in honour of Mr. A. Guillaumrs, National Museum of Natural History, Paris, to whom I am indebted for the identification of the genus.

Distirib. Endemic.

\section{(41) Terminalia carolinensis KaneHrRA sp. nov.}

Arbor $20 \mathrm{~m}$. alta $120 \mathrm{~cm}$. diametro, ramis et foliis subtus et inflorescentiis plus minusve ferrugineo-pubescentibus, ramis glabris, ramulis incrassatis ultimis circiter $1.5 \mathrm{~cm}$. diametro, cicatricibus multis magnis instructis; foliis verticillatis, coriaceis, in siccitate supra olivaceo-viridibus, glaberrimis, nitidis, subtus subferrugineo-villosis praesertim ad costam nervosque, oblongo-obovatis, 20-30 cm. longis, $10-14 \mathrm{~cm}$. latis, apice rotundatis, abrupte apiculatis, basi cuneato-acuminatis, decurrentibus, nervis primariis utrinque 18-22, prope marginem arcuato-anastomosantibus, secundariis inter primariis transversis, petiolo incrassato $5 \mathrm{~mm}$. longo, ferrugineo-pubescente ; racemis terminalibus, dioeceis(?), ad $30 \mathrm{~cm}$. longis, ferrugineo-pubescentibus, floribus masculinis pedicellatis, pedicellis tenui- 
bus, $3.5 \mathrm{~cm}$. longis, calycibus 5-lobatis, lobis acuminatis, $2.5 \mathrm{~mm}$. longis, $1.5 \mathrm{~mm}$. latis, intus basi dense hirsutis, staminibus plerumque 8 , filamentis inaequalibus, 2.5 ad $4 \mathrm{~mm}$. longis; fructibus haud stipitatis, oblongo-ellipsoideis leviter compressis, utrinque acutis, $5-7 \mathrm{~cm}$. longis, 4 cm. latis.

Kusai 1312 (fr.), 1364 (flos.), ! 1406 (fr.)! July 1931; Ponape Kolonia 1536 (fr.), Kity 1570 (fr.) Aug. 1931 (The types in Herb. Kyushu Imp. Univ.). Very common in primary forests at low altitudes in Kusai island and also found in Ponape in wet jungles at low altitudes. A very large tree with straight-boled trunk and horizontally spreading branches, leaves large and shining, whorled at the top of branches, fruit sessile oblongellipsoid, acute at both ends, somewhat compressed, 5-7 cm. long.

The present tree is very near Terminalia crassiramea Merr. from the Philippines, but is distinguished by its fewer-nerved leaves which are much more acute at the base and also by its large somewhat compressed fruits.

Distrib. Endemic, known only from Kusai and Ponape.

Nat. names: Ka (kusai), Keima (Ponape).

\section{(42) Polyscias subcapitata Kanehrra sp. nov.}

Arbuscula 1-2 m. alta, glabra, ramulis teretibus, $8-12 \mathrm{~mm}$. diametro; foliis alternis, pinnatis, usque ad $40 \mathrm{~cm}$. vel ultra longis, petiolo basi incrassato; foliolis 7-11, suboppositis, chartaceis, in siccitate olivaceopallidis, oblongis ad oblongo-ovatis, usque ad $15 \mathrm{~cm}$. longis, $8 \mathrm{~cm}$. latis, apice subacuminatis, basi plerumque inaequilateralibus, obtusis ad subrotundatis, margine remote setaceo-serratis vel integris, nervis primariis utrinque 6 vel 7, arcuato-anastomosantibus, reticulis utrinque perspicuis, petiolulis circ. $5 \mathrm{~mm}$. longis; inflorescentiis terminalibus, usque ad $35 \mathrm{~cm}$. longis, parce ramosis, tenuibus, ramis patulis, floribus umbellatim-confertis, subcapitatis circ. $20-23 \mathrm{~mm}$. diametro, pedicellis $4 \mathrm{~mm}$. longis, articulatis, calycibus infundibuliformis, $2 \mathrm{~mm}$. longis, $1.8 \mathrm{~mm}$. latis, irregulariter dentatis, stigma 5-lobata, lobis patulis, ovario 5-loculare, fructibus globosis 6-7 mm. latis, sulcatis, seminibus 3 ad 5 , reniformibus, compressis, $5 \mathrm{~mm}$. longis.

Apparently allied to Polyscias grandifolic Volkens but is distinguished by its setacous-serrate leaves and few branched inflorescences, the flowers somewhat globose umbels resembling heads at the ends of the branches, and its 5-celled fruit.

Kusai Nos. 1327! 1334, 1368, 1402 July 1931 (The type in Herb. Forest Inst. Kyushu Imp. Univ.). In primary forests at medium altitudes. 
Distrib. Endemic.

(43) Psychotria Merrillii Kanehira sp. nov.

Frutex erectus 2-3 m. altus, ramis ramulique teretibns, glabris, fuscobruneis ; foliis subverticillatis, subcoriaceis, in siccitate purpureo-brunneis, utrinque subconcoloribus subtus praesertim ad costam nervosque disperse breviter rufo-villosis vel glabrescentibus, oblongis ad obovatis, $7-13 \mathrm{~cm}$. longis, 4-6 cm. latis, apice breviter acute acuminatis, basi cuneatis, nervis primariis utrinque 8 ad 10, distinctis, circiter margine arcuato-unitis, reticulis obsoletis vel subobsoletis, petiolo crasso, usque ad $3.5 \mathrm{~cm}$. longo, glabrescente; inflorescentiis terminalibus, fasciculatis, 5- ad 8-floris, floribus longe pedicellatis, pedicellis usque ad $18 \mathrm{~mm}$. longis, tenuibus, calycibus turbinatis $2 \mathrm{~mm}$. longis, $1.5 \mathrm{~mm}$. latis, irregulariter acute denticulatis, corollae tubo $3.5 \mathrm{~mm}$. longo, extus parce puberulo, intus villoso, lobis 5 , lanceolatis, $4.5 \mathrm{~mm}$. longis, $1.5 \mathrm{~mm}$. latis, acutis, apice intus glandulosis, ovario globoso, glabro, 2-locellato ; fructibus maturis coccinis, oblongis, $10 \mathrm{~mm}$. longis, pyrenis seminibusque plano-convexis, dorso distincte carinatis.

A small tree or shrub, leaves verticellate at the top of the branchlets, oblong to obovate with stout petioles; inflorescences terminal, fascicled, long-pedicelled, fruits red when fresh. This appears to be distinct from all the species considered by VALETon in EngL. Bot. Jahrb. LXIII (1930) 313-317.

Ponape Niinoani-zan 843 Aug. 1929; Nanaraut-zan 1605, 1618, 1661 ! Aug. 1931. Named in honour of Director Merrild, New York Botanical Garden. (The type in Herb. Forest Inst. Kyushu Imp. Univ.) In dense wet primary forests at $4-500 \mathrm{~m}$. alt.

Distrib. Endemic, known only from Ponape.

(44) Rhopalobrachium megacarpus (KaNeHIRA) KaNeHIRA comb. nov.

Timonius megacarpus KanehIRA in Bot. Mag. Japan XLVI (1932) 494.

Tol, Truk, Nos. 1275! 1712, 1713, June-Aug. 1931.

For the determination of the genus, the author is indebted to Mr. A. Guillaumin, National Museum of Natural History, Paris. A genus known only from New Caledonia.

Errata

Page 452, line 15, for Horsfieldia palauense, read Horsfieldia palauensis. Page 454, line 10, for Pittosporum kusaiensis, read Pittosporum kusaiense 